\title{
User Satisfied Online IaaS Cloud Billing Architecture with the Help of Billboard Manager
}

\author{
Rajesh Bose ${ }^{1}$, Sandip Roy ${ }^{2}$ and Debabrata Sarddar $^{3}$ \\ ${ }^{1}$ Senior Project Engineer, Simplex Infrastructures Ltd., Kolkata, West Bengal, \\ India \\ ${ }^{2}$ Department of Information Technology, Brainware Group of Institutions, \\ Kolkata, West Bengal, India \\ ${ }^{3}$ Department of Computer Science \& Engineering, University of Kalyani, Kalyani, \\ West Bengal, India \\ 1'bose.raj00028@gmail.com, ${ }^{2}$ sandiproy86@gmail.com,33sarddar1@gmail.com
}

\begin{abstract}
In the context of cloud computing, no other aspect looms larger over all others than billing in Infrastructure-as-a-Service or IaaS as it is also known. This paper proposes a checklist option on IaaS Online Billing System that is described as being transparent in its computation in incorporating information on cloud resources availed and consumed, the period under consideration, and the charges accrued, thereon, for utilizing services against payment on-demand. We have discussed here, our proposed Billboard Manager with the checklist option which helps the cloud user in ascertaining how much resources have been consumed and then pay for the services enjoyed. At preset intervals of customers' choosing, a cloud service provider would be able to inform the users the actual usage. A user is required to thoroughly examine the information and approve the same, for final billing to take place. In case of discrepancy, a user would have the option to raise issues in pre-billing charge statement with the cloud service provider for mutual verification; and to arrive at the final bill. With the aid of this system, customer satisfaction could be significantly enhanced.
\end{abstract}

Keywords: Billboard Manager, Cloud service provider, Infrastructure-as-a-Service, Online billing system, Payment-On-Demand

\section{Introduction}

The phrase 'cloud computing' has grown to an almost ubiquitous stature in the context of connected network computing environments where efficiency is spoken in the same breath as cost-effectiveness. That is not to say, however, that the technology is cheap, or while being readily available off-the-shelf in most cases, not redundant or unstable. On the contrary, cloud computing is fast growing to be the backbone for small to large-scale organizations around the globe. With even individuals, entrepreneurs and start-ups eyeing their books of accounts while trying to eke out the maximum profits out of their IT investments, it is no surprise that cloud computing as we now see today, can only be expected to grow to at a rapid clip than ever before. Networking technology received a shot in the arm ever since the Internet allowed virtual doors to be opened to a worldwide audience on a hitherto unprecedented scale. Entrepreneurs and industries alike had access to information served on a platter mix of hardware and software sitting thousands and miles away. Two decades later, the advent of server farms operated by multinational software companies have made it possible to sift through trillions of gigabytes of information every single second. With data and information flowing as they were, manufacture of IT hardware saw a huge rise to meet the demands of storing and 
processing the oceans of bits and bytes traversing around the world every single second. In spite of hardware prices holding relatively steady given the exponential increase in computing power, the surge to maximize investments were becoming an overriding priority. So much so, it was not lost to businesses, small and large alike, that pooling resources through vast swathes of network-connected computers could be cheaper and less maintenance-intensive in the long-run. Thus, was born the concept of 'cloud computing'. The internet came to be known as the 'cloud'; and services offered based on it, were bracketed under the umbrella term called 'cloud computing'. The proliferation of cloud computing allowed businesses to make a lateral movement away from situations wherein large sums had to be driven into for capital expenditure, to a more 'pay-byoperating-expense' approach. IT investments began to look more promising as businesses across the globe realized that no longer did they need to invest and maintain costly hardware all by themselves. The concept of sharing pooled resources gradually gained traction as network reliability and resilience began registering uptimes close to $100 \%$. Organizations testing the hitherto uncharted waters of cloud computing now realized that to wade deeper would now require cost comparisons and weighing of benefits offered. Since, rising costs towards investments and maintenance were decisive factors in the push towards cloud computing, deep studies were conducted, and experiences shared on how best to balance resource utilizations vis-a-vis the price of staying on cloud. With the interminable pressure to maximize operating profits at every level, organizations and businesses became keenly aware to make good use of the money which they were willing to pay for services offered by cloud service providers. This demand ushered in the "pay as you go model" where users were required to make payments against billing cycles for an ordered set of cloud services enjoyed. The model which, today, has been adopted the world over, threw a slew of challenges to technology evangelists and enthusiasts alike. In the entire gamut of operations, the standout feature after security issues, remains the aspect of billing. It is this facet of operations that the pressing need for a capable billing and vetting software becomes a necessity. Primarily, cloud computing is served up in three distinct - though many consider these to be intertwined - offerings. These models, viz., software, platform, and infrastructure are accessible as service accessible via the internet. In charging users for services availed, a cloud computing model differ from web hosting in that the rates are based on usage over a period of time. The more traditional web hosting services are seldom as flexible as the former. The calculation of charges of using cloud computing services does not merely stop at time utilization. Rather, the pricing points may, and often are, subject to Quality of Service - QoS - requirements [1]. Thus, it becomes imperative to adhere to a verifiable process of billing whereby cloud service providers and their users have undistorted view of the resources utilized and the period involved in detail. A typical cloud service provider, in a commercial setting, normally undertakes the managing billing transaction and bill generation processes without any external aid or influence. This makes it difficult, if not entirely impossible, to verify consistency of billed and billable transactions to any degree of accuracy. To obtain a better understanding of the importance of billing, it is essential that the service architecture be discussed at this point. For instance, Infrastructure-as-a-Service - or IaaS, as it is commonly known - refers to a computing infrastructure encompassing networking, hardware, operating systems and software servers running in virtualized environments all offered as a service bundle. The purpose of this service bundle is to extend users the capabilities offered by the service in a manner regulated by mutually agreed policies. The QoS features are governed by these policies; in the absence of which, the very advantage offered by cloud computing models would remain obscured and enmeshed in uncertainty and doubt. The system that a cloud service offers, therefore, enables users to utilize capabilities in a consistent manner within the predetermined policies stipulated by the description of service [2]. Transparency in billing, therefore, automatically becomes of paramount importance to users. In almost all cases involving commercial offerings of 
cloud services, transactions billed and the management, thereof, are processed by the associated cloud service provider. There is, therefore, to a great degree of existence of the absence of verification of the transactions billed. IaaS, or infrastructure-as-a-service, is inclusive of networking, hardware, operating systems and software servers running on virtualized environments - packaged into a single entity and offered as a service. In situations where such services are enabled through an interface and delivered under the specifications of the associated service description [2], users would require transparency insofar as the services offered are concerned, as well as, the billing [3]. More often than not, IaaS is viewed as one of the several cloud delivery models where billing becomes a difficult task demanding resources and great degree of attention to every conceivable detail involved. As such, it may be inferred that as cloud services and infrastructure is scaled upwards, so do the complexity of billing management rises in direct proportion. Attention is focussed on billing to help add to the efficiency of IaaS cloud. To provide a handle for estimating and comparing costs, in view of the widely varying costs ranging across various infrastructure services offered by different IaaS providers, an online IaaS billing system becomes essential. This paper has been organized in a manner as follows: an overview of Cloud Delivery Model has been briefly discussed in section 2; in Section 3 discusses cloud deployment models; while section 4, elaborates on IAAS components; we have explained our proposed work in section 5; the algorithm of the checklist option has been detailed in section 6 ; the flowchart illustrating the course of our proposed work and the underlying logic is explained in section 7; our related works are discussed in section 8; and, finally, the conclusion of the paper is presented in section 9 .

\section{Cloud Delivery Models}

Cloud delivery models can be broadly segregated into the three different types. These are Infrastructure-as-a-Service, Platform-as-a-Service and Software-as-aService. These are also commonly referred to as IaaS, PaaS and SaaS, respectively as shown in Figure 1. These can be further accessed across public, private, community and hybrid, or a mixture, of clouds.

\subsection{Infrastructure-as-a-Service}

What IaaS allows to do, is to extend the reach and avail services which would otherwise be of extensively capital-intensive in nature. With IaaS, the user simply accesses services in terms of number of servers, the operating systems, the file storage systems, networking infrastructure through one common service delivery point. It can also be referred to as Hardware-as-a-Service as the hardware which would have otherwise needs to be purchased can be hired from a service provider on an on-demand basis. Almost the whole gamut of networking from IP addressed, load balancers, virtual local networks, firewalls is offered by such cloud service providers on demand. 


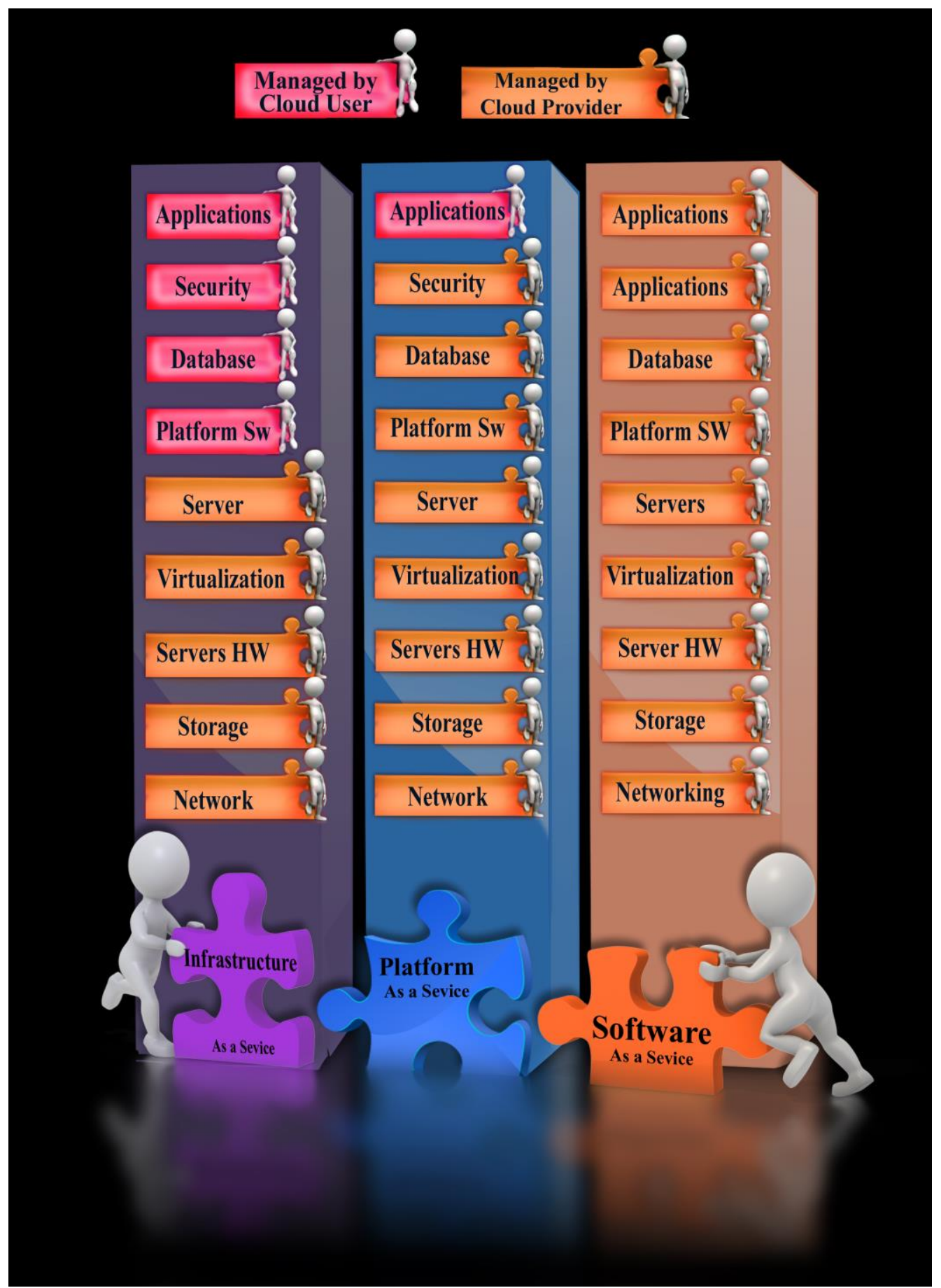

Figure 1. Cloud Delivery Model

\subsection{Platform-as-a-Service}

Typically, such a service allows developers to develop and deploy their applications without having to install, commission, manage and undertake periodic maintenance and upgrade tasks of hardware resources and software platforms. The user hiring such service can change the desired settings to suit their requirements.

\subsection{Software-as-a-Service}


Probably one of the most commonly seen applications of cloud computing delivery models is Software-as-a-Service. The users are not required at all to concern themselves with the installation, configuration and management of hardware and software needed to accomplish a given task. It is the cloud service provider who sets up the service. Examples of such are Google Docs, Microsoft Live, etc. While the advantages of such a cloud model are obvious insofar as reduced up-front costs, no apprehension or confusion about licensing norms, significant reduction of hardware resources and reduced version incompatibility issues, the issues of billing management, synchronizing the migration process of client and provider are viewed as disadvantages. In cases where a user or an enterprise uses PaaS or SaaS, the service usage logs are required for accounting the charges billed against the number of software processes and hardware resources utilized and hired from the cloud service provider.

\section{Cloud Deployment Models}

\subsection{Private Cloud}

Such clouds are maintained and operated for an organization to cater to its business needs and processes. This type of cloud can be either managed by the organization using its own dedicated resources, or by a third-party. Private cloud infrastructure may exist either on the premises or off it.

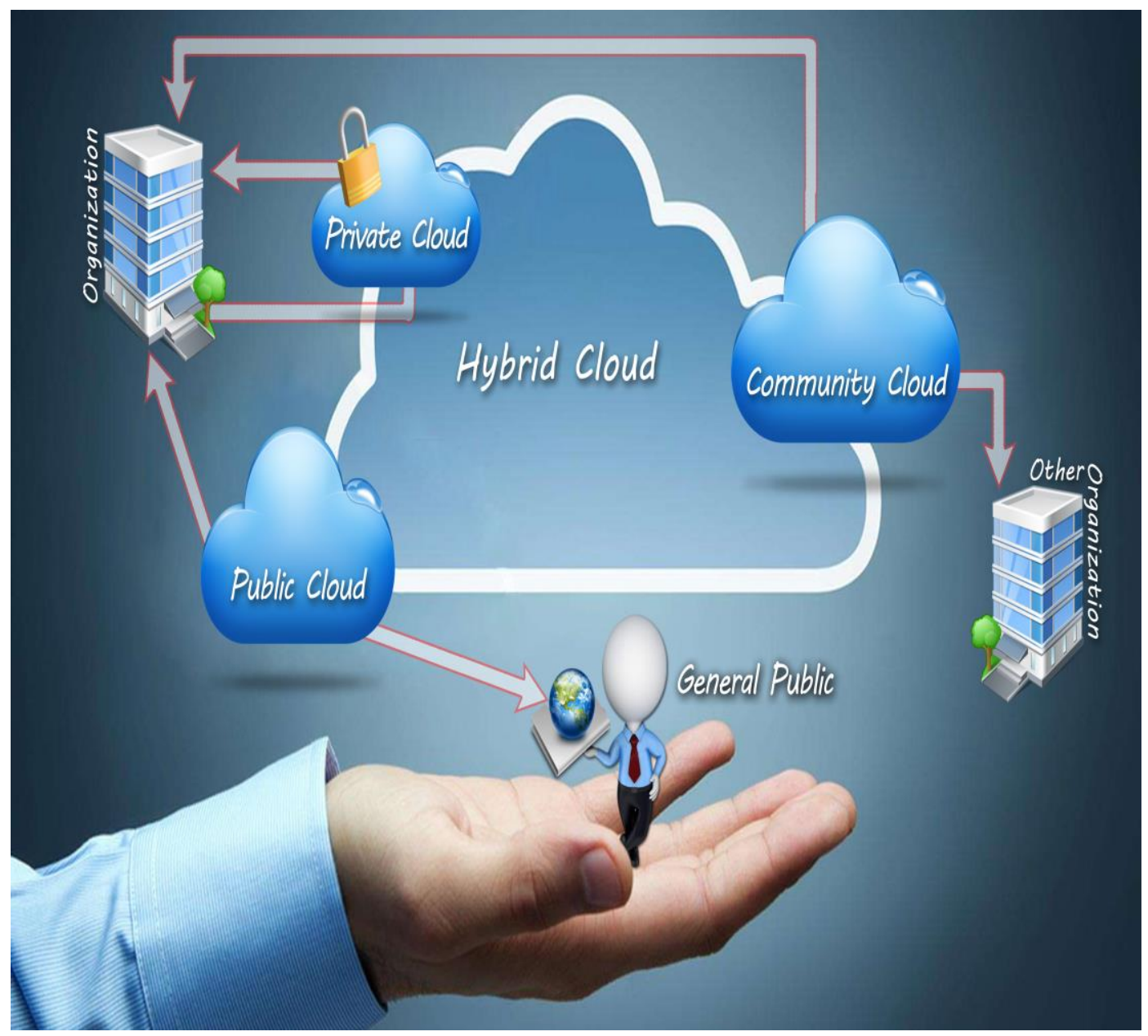

Figure 2. Cloud Deployment Models 


\subsection{Community Cloud}

As the name suggests, more than one organization participate and pool their resources to set up a cloud infrastructure that addresses their joint concerns. This could be missionspecific, to meet security requirements, comply with legal or statutory policies). Community clouds can be managed by organizations at their premises or off it, or by a third-party.

\subsection{Public Cloud}

A public cloud is one, the services of which, the general public or businesses can hire. Usually, public clouds are operating by large business or industry groups specifically in the market for selling services deployed over cloud.

\subsection{Hybrid Cloud}

As the name suggests, hybrid clouds offer a mix of two or more clouds which could be private, public or community based. The entities, however, are linked to each other by technology standards that allow for portability of data and applications. An example worth considering would be cloud bursting for load-balancing between clouds.

\section{IaaS Components}

As a form of hosting, Infrastructure-as-a-Service does not differ much from the traditional concept. Network access, routing services and storage are offered as standard features. The IaaS provider supplies the hardware and the associated platform overall managed by the provider itself, needed to operate applications and store data. Bandwidth, memory and storage allocations on an on-demand basis are normally included. Almost all vendors vie for cornering the largest possible market share by promoting performance of their cloud offerings vis-a-vis costs. Services to a typical IaaS pack can be purchased either for a period of contract, or paid for specific resources used in a given period of time. The latter also referred to in common parlance as "pay-as-you-go" basis. Figure 3 shows the Logical component of Billboard Metering Infrastructure-as-a-Services. IaaS components include:

\subsection{Storage Services}

Cloud storage implies and refers to storing of data across pools of servers connected by intricate layers and points connected over networks. These could either be over the Internet, or across private networks. Though, the former is always implied when cloud storage is discussed. Rapid accessibility, greater degree of reliability and considerable ease in terms of data backup, archival, and disaster recovery management, make cloud storage an attractive proposition when the added factors of not having to purchase, maintain, manage and conduct periodical upgrades to expensive hardware are also taken into account. As a result, cloud storage has been found to have an effect on reducing overall storage costs. However, these do not necessarily translate to having reduced security and compliance issues. Adequate measures are often needed to be put in place to ensure that security and data integrity is not compromised. The benefits of cloud storage become immediately obvious. With the help of the Internet, files stored can be accessed from anywhere in the cloud. Further, the distribution and copies of data stored off-site allows for decreasing the overheads associated with disaster recovery. 


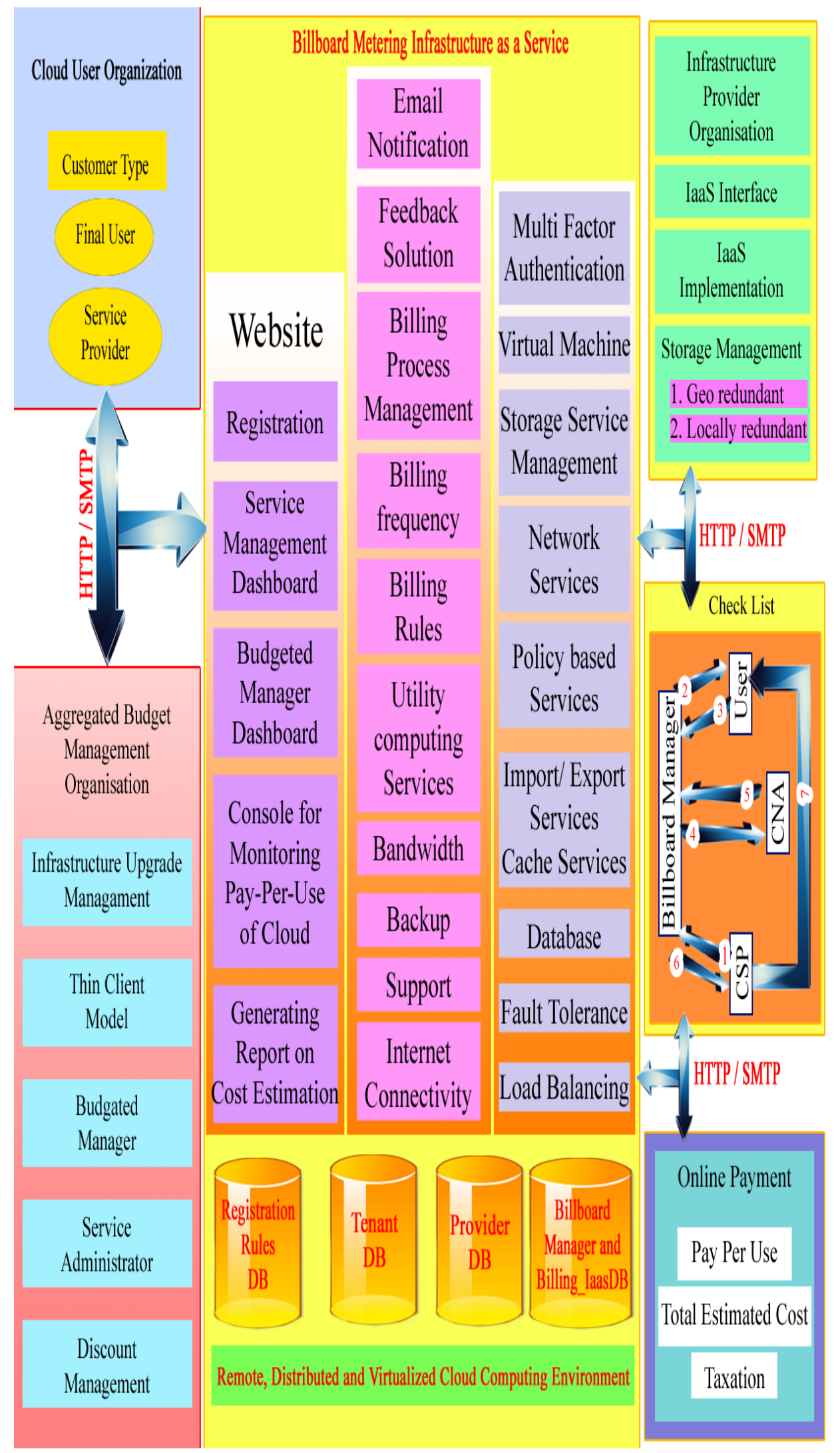

Figure 3. Logical component of Billboard Metering Infrastructure as a Service 


\subsection{Database Services}

Similar to cloud storage services, a database is made accessible to clients over the cloud. As on demand, users can access the database over the Internet to connect the cloud database service provider's servers. Databases hosted on the cloud are able to gain advantage over the traditional models, by harnessing effective resource allocation, optimized and dynamic scaling, multi-tenancy and high-availability. Added advantages include automatic failover, automated recovery from failures, dynamic scaling, and minimum investment. All these translate to better maintenance opportunities and assurance of better performance gains. Again, as in storage model, secure and privacy issues are the key overriding concerns which preclude most businesses to shy away from this model. Further, should there be a critical failure, or winding up of the business of the cloud database service provider, vital business information might be irrevocably lost. A possibility that key decision makers are always forced to consider prior to considering moving even a part of database resources over to a cloud service provider.

\subsection{Cache Services}

With the aid of a caching service, an application is able to call upon in times when memory is needed to be elastic for increasing performance. This helps to divert the extensive demand loads placed on the data tier so that the given application is able to generate more throughput. Latency times can see remarkable improvements as caching services offered on the cloud are markedly faster than disk-based database systems.

\subsection{Recovery Services}

As part of the system of naming in the field of cloud computing, Recovery-as-aService or RaaS is also considered to be a part along with IaaS, PaaS and SaaS. Alternately referred to as Disaster Recovery as a Service or DRaaS, this is categorized as being used with the intention of providing a means to safeguard an application or data from disasters - either natural or man-made. Usually such protection is extended by means of activating full recovery mode in the cloud. By providing protection to data and extending standby capacity on-demand to aid in quick recovery of application, RaaS differs from other cloud-based backup offerings. In a typical scenario, RaaS is delivered in a cloud computing model which allows payment only when recovery is made. This makes it more efficient than a traditional recovery system where resources must be operating at all times. IaaS offers a platform for running virtual systems that can be accessed in the cloud. The technique usually involves creation of virtual machines that are installed with the software necessary, and configured to be operated and accessed across the Internet, or over a network. Once a virtual machine is prepared, it is uploaded onto the hosting platform of the IaaS where it is configured to utilize the resources of the vendor's storage devices. Upon configuration, the virtual machine can be deployed and initiated via an automation process designed to automatically locate the available hardware on which to run the virtual machine. The hardware - consisting of computers and storage devices needed for the application are owned and managed by the IaaS provider. All is left to the customer, therefore, is to monitor all custom and licensed software for any unexpected failures that may occur. In this context, IaaS may be viewed as an option that not only accords flexibility but happens to be the leading choice for shifting applications to the cloud when time is a constraint insofar as reworking an application's code for a cloud deployment is concerned.

\subsection{Virtual Machine Services}

Riding on the crest of the ever-growing popularity of Infrastructure-as-a-Server, the concept of hosting virtual desktops and extending services so marketed under the 
nomenclature of Desktop-as-a-Service found a great deal of acceptance in a short span of time. It is a form of computing services which allow businesses to provide, extend accessibility, operate and withdraw virtual desktops to users. Like the similar IaaS model, payments are made for services consumed. Enterprises can choose to establish connection supported by private networking backbone instead of utilizing the internet. Commonly, the provider extending the DaaS would offer to host the virtual computers, ensure security and protection of data, and regulate the amount of data flowing to guarantee uptime. Almost all known solutions are equipped with a portal which allows businesses right from management till billing. Desktop-as-a-Service is a mode to ensure that sufficient resources insofar as desktop environments are concerned, exist and are available for new users. Since, these virtualized desktops can be projected over the internet; users can easily log in and have their own tailored workspaces active and ready for use almost anywhere with internet connectivity.

\subsection{Policy based Services}

Policy-based services offer automated processes which can be tweaked and regulated by organizations. Such services have a direct bearing on policies, as defined by an enterprise, on infrastructure services accessible to an individual user or user groups.

\subsection{Networking-as-a-Service}

The latest among cloud services - Network-as-a-Service allows businesses to extended and support virtual networks. NaaS includes features which include firewalls, load balancing, and acceleration services on wide area networks. It aims at providing a synchronized connection between storage medium, networking equipment, and servers all of which act in concert dynamically to address the requirements of a virtual infrastructure. Yet in another cases as well, NaaS is able to support QoS factors and various auditing and monitoring services extended over a network. By itself, NaaS does not require upfront investments to be made and is able to sustain scalability issues, while remaining robust and flexible, and uncompressing in security aspects.

\subsection{Multi-factor Authentication Services}

This form of system involves the practice of more than one authentication process to verify whether a transaction can be deemed legitimate. The primary objective of multifactor authentication service is to create a defensive perimeter which is composed of several layers each of which designed to thwart any unauthorized access directed at breaking in into a network or a computer system.

\subsection{Backup Services}

Much like regular backup software, online backup services have been designed to offer identical features at a considerably lower ownership cost. While data transmission over the internet may be viewed as being insecure, the storage of data in servers maintained at data centers which are operated by dedicated professionals on a round-the-clock basis is secure and closely guarded away from prying eyes. The primary advantage of using such cloud based backup services is that the chances of loss of data owing to natural calamities, accidents or incidents man-made or otherwise, are almost negligible.

\subsection{Service Level Agreement (SLA)}

As a strategy for alteration in cloud services offered, and as a bar that sets the levels of assumptions involving both the service provider and the consumer of cloud-based products, Service Level Agreements serves to act as a contract and a form of a guarantee 
aimed at allaying fears or apprehensions that a consumer might have insofar as the quality and quantity of services that can be offered in a given tenure.

In order to maintain consistency in effectively formulating a SLA, the following list of requirements needs to be implemented and asserted. The details are as follows:

- Availability at a level of $99.99 \%$ during workdays as well as on nights and in weekends.

- Fast response times.

- Data encryption for those stored as well as transmitted such that security and privacy remain uncompromised.

- Contingency plans for disaster recovery and to ensure data redundancy in order to maximize chances of recovery of data stored.

- Positioning of the data in a given locale such that it consistently meets legislation norms of the given region.

- Data accessibility and consistently high standard of retrieving data in a legible format and/or in a manner that is readable on expected lines.

- Data portability from one service provider to another.

- A well-designed process to address problems and reach resolutions consistently and accurately in the shortest possible time. For example, a call center.

- Process to address change management which would include changes made, updates incorporated and or entry of new services.

- A hierarchical process to settle disputes. This could be in the form of an escalation matrix.

- An exit strategy aimed at ensuring that the service provider can deliver smooth transition within acceptable parameters.

Service Level Agreements by their very definition are dependent on the services which they relate to. As such, with changing levels of services, SLAs need constantly be revalued and reassessed. In cloud service offerings, SLAs are invaluable as these act as standards and assist in handling issues. Many are of the opinion that SLA is a tool to safeguard the interests of a business and its assets. Further, the existence of SLA also helps in reducing severance costs and charges should a situation come to pass that the contract between the business and the cloud service provider needs to be terminated or no longer renewed. In such a situation, it becomes imperative that both parties involved, need to amicably resolve the differences and move ahead in diverging ways without incurring legal costs and charges which are ultimately a drain on resources as well as time.

\subsection{Utility Computing Service}

The idea of utility computing has existed for some time. In a grid computing deployment, this concept has often been an important hub. A precursor to cloud computing model, utility computing service packs several assets and services and offers those on a basis of payment as per consumption. As would be readily evident, the strength of this model lies in two areas, viz., reduction of overall cost, and scalability. Utility computing service has to, of course, surmount the hurdles posed by cloud computing. In a form of pyramid structure offering cloud services; for example, Amazon provider's services that are metered to second level providers who go on to extend those services to each of their clients. In such multiple layers, complexity rapidly raises requiring intense efforts to manage by service providers at all levels. An example of such system, the Amazon Dev. Pay 5, equips second level service providers with tools to quantify and proportionately bill charges for utilization of AWS services. The users can then be billed in accordance with the SLA and agreed tariff. Possibility the biggest challenge is the fact that utility computing services are viewed as attractive targets by malicious attackers. Often a potential threat can be in the form of inflated unmanageable billed charges of a business consuming a utility computing service. It is up to the service provider to secure 
the system in a manner such that malicious attacks are not able to access services without legally entering into a contract.

\section{Proposed Work}

Our proposed online billing system of cloud IaaS model is based on the ability of the Billboard Manager to store the Service Level Agreement (SLA), collect the bill generated from the cloud service provider (CSP) and send this to the email address of the customer enjoying the relevant services offered by the CSP. In case, the customer confirms the charges as raised by the CSP, he/she needs to approve it and notify the Billboard Manager. The Billboard Manager would then send both the SLA and the bill copy as approved by the customer, to a Cloud Node Authority (CNA) or any such other approved third-party with the appropriate legal standing and authority to arbitrate in matters of such billing. It would be the job of such CNA, or third-party, to verify the bill in the context of the SLA. Should there be no discrepancies or issues in this process, the CNA would notify its approval to the Billboard Manager. On receipt of this approval, the Billboard Manager would inform the CSP to generate the final bill. The CSP, in turn, after receiving the confirmation from the Billboard Manager, would raise the final bill and send this to the customer for online payment. Figure 4 shows the block diagram of the above matter.

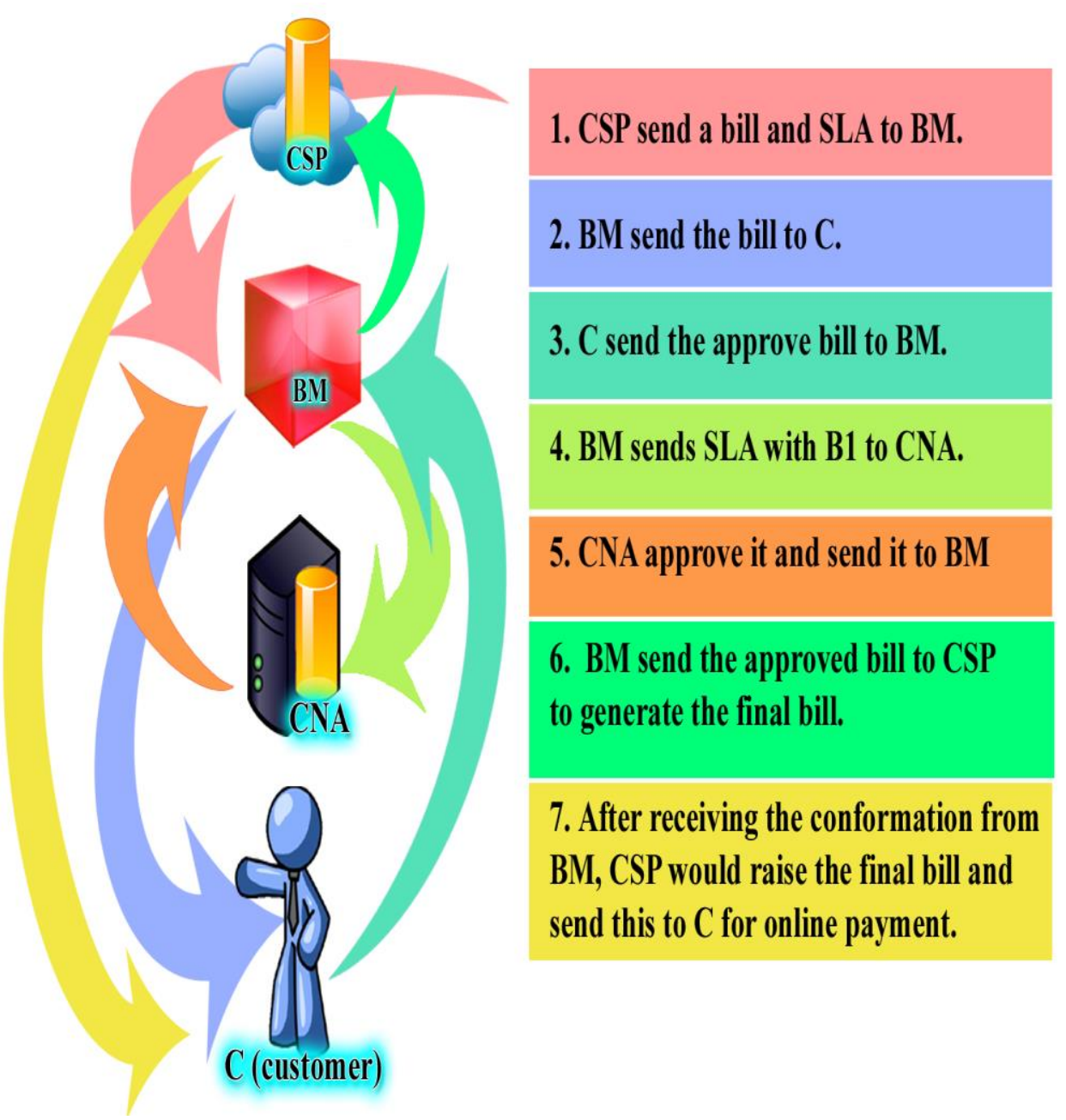

Figure 4. Block Diagram of Checklist Option for Billing Architecture with users' Satisfaction 
However, in case the user at this stage finds an error or mismatch in the usage details in the final bill so raised by the CSP, the matter would be referred to the Billboard Manager for inspection. The Billboard Manager would forward this bill, along with the comments of the user, to the CSP for conducting a re-check. In case errors are observed, the CSP would generate a corrected bill and send it to the Billboard Manager which, in turn, would forward it to the concerned user for confirmation. The user, after confirming that all errors have been addressed and corrected figures are in place, would need to send a confirmation to the Billboard Manager. At this stage, the Billboard Manager once again sends both the SLA and the now corrected bill to the CNA for necessary approval. Should there be no discrepancies found, the CNA will then approve of this bill and return it to the Billboard Manager. Finally, the Billboard Manager sends this bill to the CSP for raising the final bill bearing the sign and seal of the CNA, which is then sent to the user for payment. Figure 5 shows the block diagram of the above matter.

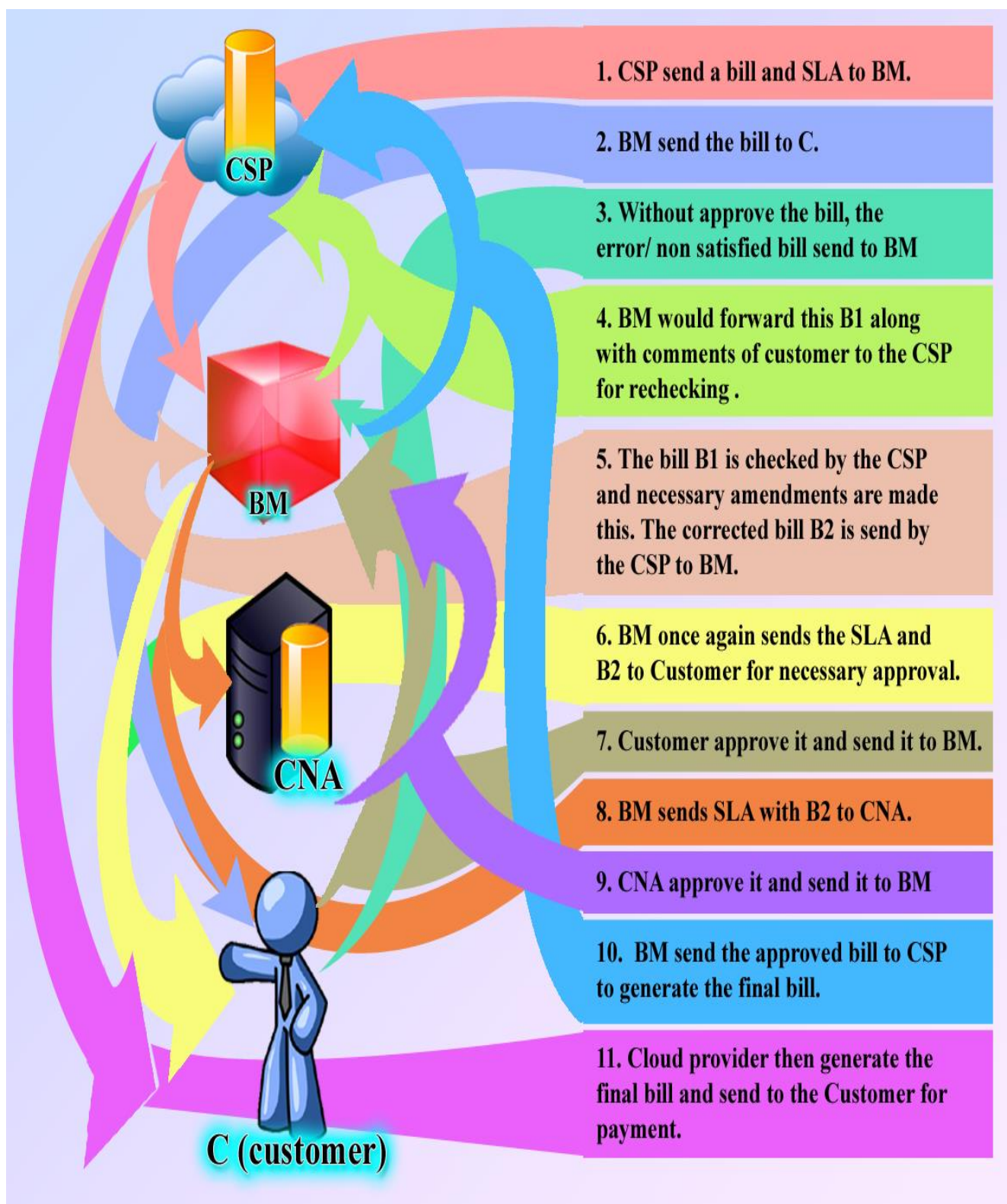

Figure 5. Block Diagram of Checklist Option for Billing Architecture without users' Satisfaction 


\section{Algorithm of Checklist Option}

Online Billing System of Cloud IaaS Model

BM - Billboard manager

CSP - Cloud Service Provider

CNA - Cloud Node Authority

SLA - Service Level Agreement

1. CSP generates a bill (denoted as 'B' for the purposes of this document) for services availed by a customer (hereinafter referred to as ' $\mathbf{C}$ ' in this document).

2. CSP sends SLA to BM.

3. $\mathrm{BM}$ collects $\mathbf{B}$ generated by the CSP.

4. BM sends $\mathbf{B}$ that is generated by CSP to the email address of $\mathbf{C}$.

5. IF $\mathbf{C}$ confirms the charges raised by the CSP:

5.1 Approves the $\mathbf{B}$.

5.2 C notifies BM.

5.3 BM sends SLA and the approved B (hereinafter referred to as 'B1' in this document) to CNA or such other recognized and appointed third-party.

5.4 CNA, or the appointed third-party, receives B1 and verifies it on the lines of the SLA to check for inconsistencies and/or errors.

5.5 After that, the CNA would notify its approval to the BM. On receipt of this approval,

5.6 BM receives the approval and informs the CSP to generate the final bill.

5.7 After receiving the confirmation from BM, CSP would raise the final bill and send this to $\mathbf{C}$ for online payment.

6. ELSE

6.1 C Finds an error or mismatch in the usage details in the bill B which is raised by the CSP.

6.2 The matter would be referred to BM for inspection.

6.3 BM would forward this B1 along the comments of the user to the CSP for rechecking.

6.4 The bill B1 is checked by the CSP and necessary amendments are made in this. The corrected bill so prepared (hereinafter referred to as 'B2' in this document) is sent by the CSP to BM.

6.5 BM once again sends the SLA and $\mathbf{B} 2$ to CNA for necessary approval.

6.6 Go to Step [5.1], [5.6], [5.7]

7. IF C paid the bill,

7.1 Payment is initiated and bill is settled.

8. ELSE

8.1 BM sends out reminders to $\mathbf{C}$ for settling the payment, after a set interval of time (hereinafter referred to as ' $\mathbf{t}_{\mathbf{1}}$ ' has elapsed. After a set count of $\mathbf{t}_{\mathbf{1}}$ intervals, if $\mathbf{C}$ has not settled the final bill, BM signals CSP so. Thereafter, the owner is on the CSP to either cease services to $\mathbf{C}$ or take any other appropriate step. 


\section{Flowchart of our Proposed Algorithm}

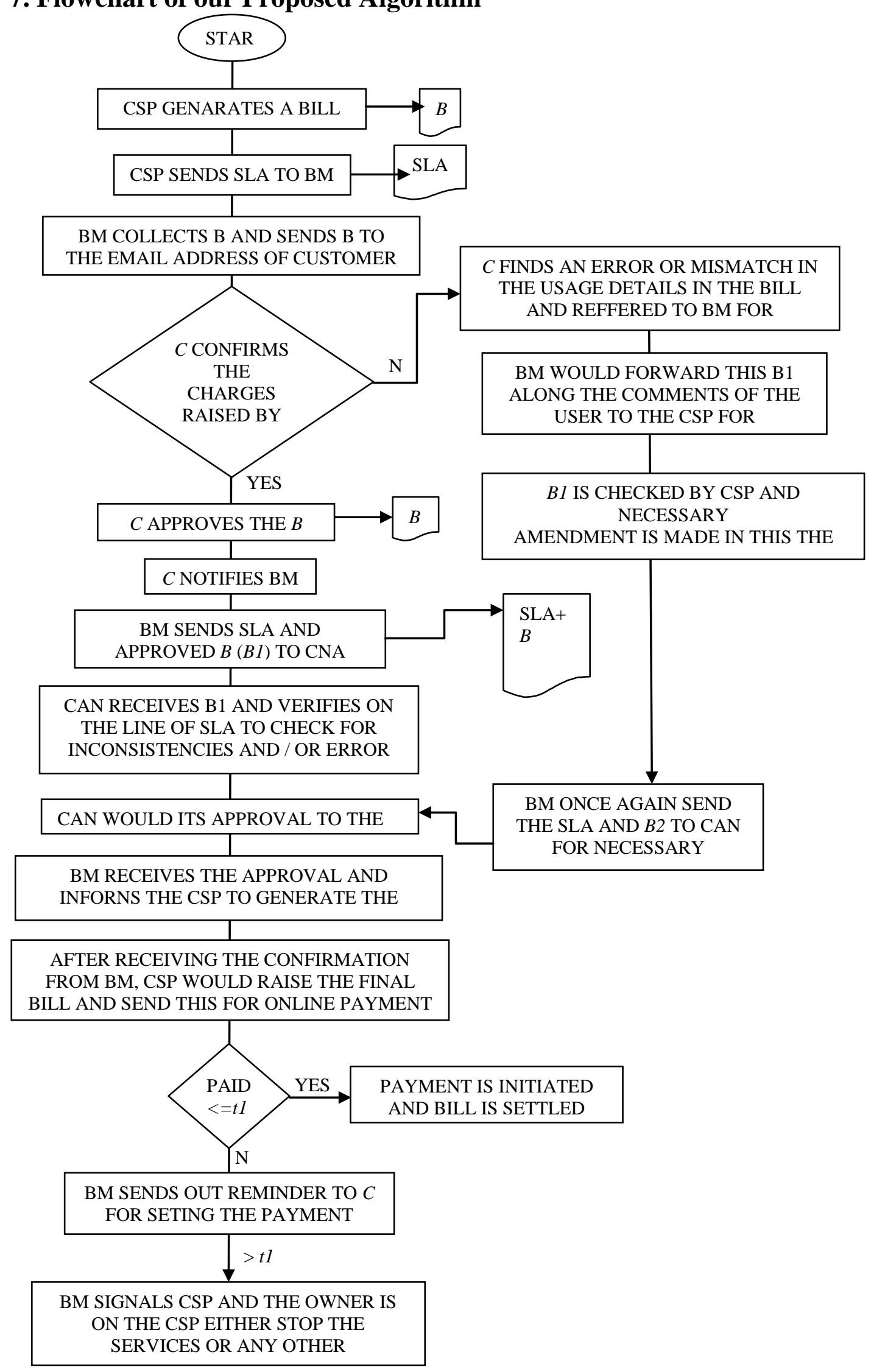




\section{Related Works}

A lot of research has been done in the field of Billing management of IaaS in cloud computing, A complete outline on various researches and trends in IaaS Billing has been presented in [5]. The authors discuss here IaaS Online Billing System that describes transparency of consumption, billing and frequency of usage of services for a cloud based pay per use system. This system describes various interactions of network and user interface. The billing rules have been stored in a database. The model has been verified through UML that demonstrates that IOBS is effective in improving user interaction by reducing time and increasing customer satisfaction. As per Luis M. Vaquero [6], the employment of advanced billing mechanisms allowing for a pay-per-use model for shared multitenant resources, the simplified programming mechanisms (Infrastructure), etc., are some of the most relevant features. Stefan Tai and other researchers introduced that the consumers of Cloud services are charged based on actual service usage. But what are appropriate costs, pricing and billing models from both consumer and service provider viewpoints? How do we calculate costs, compare alternatives, estimate risks and determine value of traditional versus cloud computing solutions? With the help of this approach we cannot estimate the cost [7]. Ang Li and others [8] proposed an approach in which the cost should be calculated per operation only not pay per use. Matthew Wachs and co-workers addressed the method that generates bill only for storage resources [9]. Maik Lindner and his workmates described an approach in which only service and infrastructure can interact [10]. Yousef, et al., [11] described the three pricing models that are used by cloud service providers for billing used resources, namely tiered pricing, perunit pricing and subscription-based pricing. Aquarium's cost policies that are assigned to resources map exactly to Yousef's pricing models. In fact, most offerings by public IaaS providers, including Amazon and Azure, offer services charged according to Yousef models. Work on resource accounting and billing has been carried out in the context of cloud federation [12-14] and (earlier) grid federation projects. The Reservoir project investigated the use of service level agreements [12] for resource provisioning in federated cloud scenarios. Georgios Gousios and others also proposed the design and implementation of Aquarium, extensible billing service software [15]. This approach provides portability but desired performance cannot be achieved. In [16], the authors proposed a secure and non-obstructive billing system called THEMIS as a remedy for the above mentioned limitations. The system uses a novel concept of a cloud notary authority (CNA) for the supervision of billing. The CNA generates mutually verifiable binding information that can be used to resolve future disputes between a user and CSP in a computational efficient way. Further scalability and fault tolerance is done in banking side by providing security for bill payment which is a web service. This leads to faster time to market, minimal computational cost, accurate, consistent and competitive pricing.

\section{Conclusion}

The objective of this paper of discussing an IaaS Online Billing System for cloud computing is to lay forth a mechanism whereby complexity is reduced while improving user interaction and response times to assure user satisfaction. Our paper is an endeavour to simplify the process of billing of IaaS services availed by individual customers without compromising on the transaction times involved in settling invoices raised by cloud service providers. With the aid of the proposed checklist option invoked through the Billboard Manager, a cloud user is armed with a tool which allows to breakdown every price component billed for IaaS services availed. Further, the proposed Billboard Manager would also enable the cloud service provider to inform the customers with accumulated billing costs at regular intervals so set by the customers themselves. While IaaS allows entrepreneurs and organizations to significantly reduce their exposure to costly outlay in procuring high-value infrastructure, without a billing system, such as our proposed 
Billboard Manager - which not only allows for cross-checks by all parties concerned, the advantage of IaaS over traditional capital investments would progressively fade.

\section{References}

[1] R. Buyya, C. S. Yeo and S. Venugopal, "Market-Oriented Cloud Computing: Vision, Hype, and Reality for Delivering IT Services as Computing Utilities", Proceedings of the 10th IEEE International Conference on High Performance Computing and Communications, (2008), pp. 9.

[2] B. Len, C. Paul and K. Rick, "Software Architecture in Practice", Pearson Education, 2nd Ed, (2003).

[3] B. P. Rimal, A. Jukan, D. Katsaros and Y. Goeleven, "Architectural Requirements for Cloud Computing Systems", An Enterprise Cloud Approach, Springer Science Business Media B.V. 2010, J Grid Computing, vol. 19, no. 3, (2011), pp. 26.

[4] Y. Jadeja and K. Modi, "Cloud Computing-Concepts, Architecture and Challenges", IEEE, (2012), pp. 4-12.

[5] S. Singh and I. Chana, "Advance Billing and Metering Architecture for Infrastructure as a Service", International Journal of Cloud Computing and Services Science (IJ-CLOSER), vol. 2, no. 2, (2013), pp. 123-133.

[6] L. M. Vaquero, L. Rodero and R. Buyya, "Dynamically Scaling Applications in Cloud", ACM SIGCOMM Computer Communication Review, vol. 41, no. 1, (2011), pp. 45-52.

[7] S. Tai, J. Nimis, A. Lenk and M. Klems, "Cloud Service Engineering", Proceedings of the 32nd ACM/IEEE International Conference on Software Engineering, vol. 2, (2010), pp. 475-476.

[8] A. Li, X. Yang, S. Kandula and M. Zhang, "CloudCmp: Comparing Public Cloud Providers", ACM, (2010), pp. 1-14.

[9] M. Wachs, L. Xu, A. Kanevsky and G. R. Ganger, "Exertion-based billing for cloud storage access", Proceeding Hot Cloud'11 Proceedings of the 3rd USENIX conference on Hot topics in cloud computing, (2011), pp. 1-5.

[10] M. Lindner, F. Galan, C. Chapman and S. Clayman, "The Cloud Supply Chain: A Framework for Information, Monitoring, Accounting and Billing", 2nd International ICST Conference on Cloud Computing, (2010), pp. 1-22.

[11] L. Youseff, M. Butrico and D. Da Silva, "Toward a Unified Ontology of Cloud Computing", In Grid Computing Environments Workshop, IEEE, (2008), pp. 1-10.

[12] E. Elmroth, F. Marquez, D. Henriksson and D. Ferrera, "Accounting and billing for federated cloud infrastructures", In Eighth International Conference on Grid and Cooperative Computing, IEEE, (2009), pp. 268-275.

[13] R. M. Piro, A. Guarise and A. Werbrouck, "Price-sensitive resource brokering with the Hybrid Pricing Model and widely overlapping price domains", Research Articles: Concurrency and Computation Practice and Experience, vol. 18, no. 8, (2006), pp. 837-850.

[14] B. Rochwerger, D. Breitgand, E. Levy, A. Galis, K. Nagin, I. M. Llorente, R. Montero, Y. Wolfsthal, E. Elmroth, J. Caceres, M. Ben-Yehuda, W. Emmerich and F. Galan, "The Reservoir model and architecture for open federated cloud computing", IBM Journal of Research and Development, vol. 53, no. 4, (2009), pp. 4:1-4:11.

[15] G. Gousios, C. K. K. Loverdos, P. Louridas, N. Koziris, "Aquarium: An Extensible Billing Platform for Cloud Infrastructures", https://code.grnet.gr/attachments/download/.../Aquarium-paper.pdf.

[16] U. Yamini and K. S. Priya, "Trusted SLA Monitoring for Billing System in Public Cloud computing Environment”, IJMER, pp. 35-41.

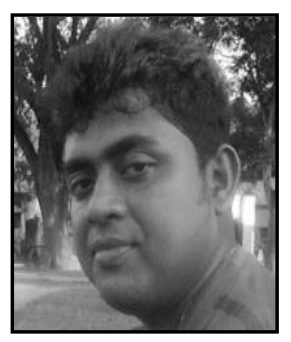

Rajesh Bose, is currently pursuing Ph.D from University of Kalyani. He is an IT professional employed as Senior Project Engineer with Simplex Infrastructures Limited, Data Center, Kolkata. He received his degree in M.Tech. in Mobile Communication and Networking from WBUT in 2007. He received his degree in B.E. in Computer Science and Engineering from BPUT in 2004. He has also several global certifications under his belt. These are CCNA, CCNP-BCRAN, and CCA (Citrix Certified Administrator for Citrix Access Gateway 9 Enterprise Edition), CCA (Citrix Certified Administrator for Citrix Xen App 5 for Windows Server 2008). His research interests include cloud computing, wireless communication and networking. 


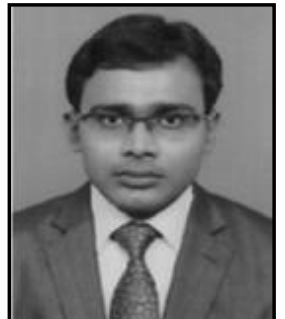

Sandip Roy, is currently pursuing Ph.D from University of Kalyani. He is an Assistant Professor in the Department of Information Technology, Brainware Group of Institutions, Kolkata, West Bengal, India. He has completed M.Tech in Computer Science \& Engineering from HIT under WBUT in 2011. He has also done his B.Tech in Information Technology from WBUT in 2008. His main areas of research interest are Cloud Computing, Data Structure and Algorithm.

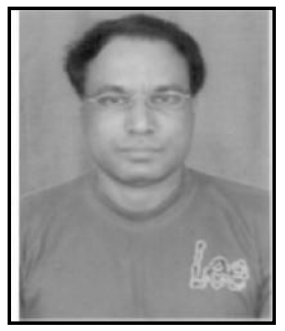

Debabrata Sarddar, is an Assistant Professor in the Department of Computer Science and Engineering, University of Kalyani, Kalyani, Nadia, West Bengal, INDIA. He has done PhD at Jadavpur University. He completed his $\mathrm{M}$. Tech in Computer Science \& Engineering from DAVV, Indore in 2006, and his B.E in Computer Science \& Engineering from NIT, Durgapur in 2001. He has published more than 75 research papers in different journals and conferences. His research interest includes wireless and mobile system and WSN, Cloud computing. 
International Journal of Grid Distribution Computing

Vol.8, No.2 (2015) 